\title{
Design and Analysing the Various Parameters of CMOS Circuit's under Bi-Triggering Method Using Cadence Tools
}

\author{
A. Sridevi ${ }^{1}$, V. Lakshmiprabha ${ }^{2}$, N. Prabhu ${ }^{1}$ \\ ${ }^{1}$ Department of ECE, SNS College of Technology, Coimbatore, India \\ ${ }^{2}$ Government College of Technology, Coimbatore, India \\ Email: profvlp@gct.ac.in
}

Received 28 March 2016; accepted 15 April 2016; published 28 July 2016

Copyright (C) 2016 by authors and Scientific Research Publishing Inc.

This work is licensed under the Creative Commons Attribution International License (CC BY). http://creativecommons.org/licenses/by/4.0/

(c) (i) Open Access

\section{Abstract}

Reducing the power and energy required by the device/circuit to operate is the main aim of this paper. Here the new design is implemented to reduce the power consumption of the device using the triggering pulses. The proposed triggering method uses a complementary MOS transistor (pMOS and nMOS) as a voltage divider and ground leakage suppressor (i.e.); these designs are named as Trig01 and Trig10 designs. In Trig01 design the pair of CMOS is placed in the voltage divider part; similarly in Trig10 design the pair of CMOS is placed at the ground leakage suppressor part. Standard CMOS gates like NOT, NAND, NOR, EX-OR etc. are designed with these technologies and these gates are designed with $180 \mathrm{~nm}$ technology file in the cadence tool suite; compared to the normal CMOS gates, the Bi-Trig gate contains 4 inputs and 2 outputs. The two extra inputs are used as Bi-Trig control signaling inputs. There are 2 control inputs and thus $2^{2}=4$ combination of controlling is done (i.e.); both pMOS and nMOS are ON, both pMOS and nMOS are OFF, pMOS ON and nMOS OFF and pMOS ON and nMOS ON. Depending on the usage of the circuit, the mode of operation is switched to any one of the combination. If the output of the circuit is not used anywhere in the total block, that specified circuit can be switched into idle mode by means of switched OFF both the pMOS and nMOS transistor in the control unit. This reduces the leakage current and also the power wastage of the circuits in the total block. Bi-Trig controlled circuit reduces the power consumption and leakage power of the circuit without affecting a performance of the circuits.

\section{Keywords}

Bi-Triggering, Power Analysis, Energy Analysis, Circuit Simulation, Delay Analysis, Sub Clock Method 


\section{Introduction}

Leakage power occurs due to the error voltage in the transistor during the ideal mode. This leads to increase of the power consumption and also the energy, and to reduction of the power wastage and consumption. The proposed method is used. To obtain the correct result with reduced power consumption the Bi-Trig is used.

In previous method i.e.., Dual Mode logic [1], the operation is determined as dynamic and static function. The performance of the circuit is high only in dynamic mode; it is proved in the paper [1]. Thus similarly the paper [2] tells about the low-power dynamic operation method, in which the width of the transistor is tuned, and it reduces the overall power. This gives the result of about $25 \%$ of power consumption reduction.

The main drawback of the paper [2] is that the circuit does not have a driving load capacity and thus it is overcome by using the nanometer technology as given in the paper [3]. To overcome the drawback of the subthreshold DML [1]-[4], this proposed system is designed with two new methodologies.

Trig01: pMOS and nMOS are connected at the voltage side of the standard CMOS circuit.

Trig10: pMOS and nMOS are connected at the ground leakage suppressor side of the standard CMOS circuit.

pMOS and nMOS are used as a Bi-Trig Control circuit to drive the entire circuit by means of voltage divider method. In design-1 the control circuit is connected at the voltage divider side of the circuit to control the entire circuit by separating the given supply voltage (VDD). Similarly in the second design methodology the Bi-Trig Control circuit is placed at the ground leakage suppressor side in order to reduce the leakage current through the circuit to ground terminal.

\section{Proposed System}

The design developed to reduce the leakage power in CMOS circuit during the active mode of operation. By activating the CMOS transistor there will be a direct connection between the VDD and ground will happen at the 0.7 voltage switching [5]. This causes a shot link to ground leakage current will more at the time of switching [6]. In order to reduce the leakage current the Bi-Trig method is implemented. There are two design method is implemented in order to rectify the problems. Bi mode is used to reduce the ideal current and full mode operation is used to drive the large capacitance load without using the cascading inverter units.

\subsection{Trig01}

In this schematic methodology the control signal is located at the voltage divider side of the CMOS circuit. By this process the operating voltage which is given to the circuit is divided into two parts by using the nMOS and pMOS transistor. These transistors are connected in with two separate inputs as shown in the Figure 1 . Thus the input is given directly to the CMOS circuit and the output is forwarded directly from the circuit. For half mode power output is control by the Bi-Trig control circuit. (i.e.) Either pMOS is ON or nMOS is switched ON. pMOS is switched ON and OFF using the trig pulse as 0 and 1 . Similarly the nMOS is switched ON and OFF by using the control signal as 1 and 0 . For a full mode operation in order to drive the full mode the control circuitry is switched ON fully mode i.e., pMOS and nMOS both are in closed loop. Thus the full power is given to the CMOS circuit.

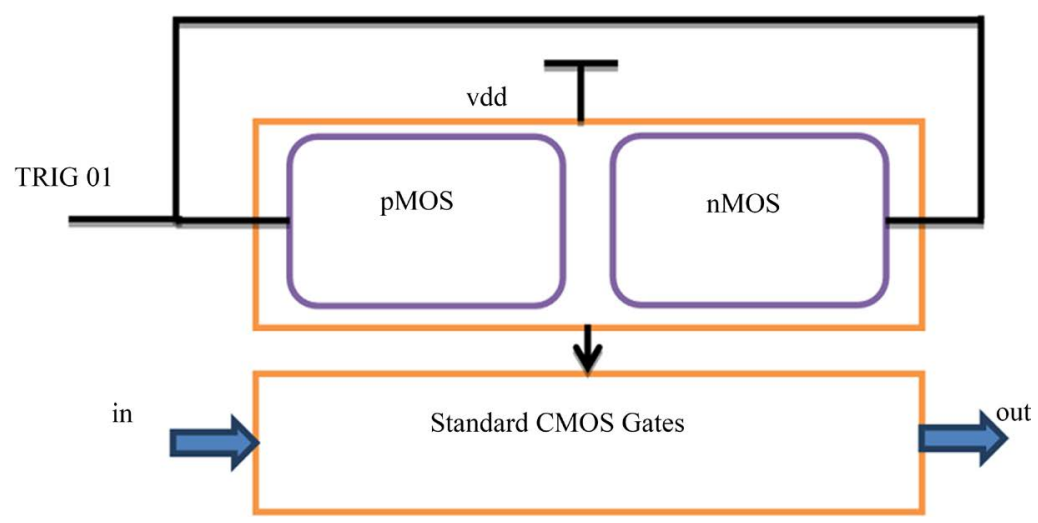

Figure 1. Design Trig01 block diagram. 


\subsection{Trig10}

In this schematic methodology the control circuit is placed at the ground leakage suppressor side of the CMOS circuit. By this technique the error current due to the short circuit between the VDD and GND is reduced by using the nMOS and pMOS transistor. These transistors are connected in with two separate inputs as shown in the Figure 2. Thus the input is given straight to the proposed circuit and the output is drive directly from the circuit.

For half mode power output is control by the Bi-Trig control circuit. (i.e.,) Either pMOS is ON or nMOS is switched ON. pMOS is switched ON and OFF using the trigger pulse as 0 and 1 . Similarly the nMOS is switched ON and OFF by the trigger pulse as 1 and 0 . For a full power operation to drive the large load the trigger circuitry is switched ON fully mode i.e., pMOS and nMOS both are in closed loop. Thus the full power is given to the CMOS circuit.

\section{Implementation of the Design in Basic CMOS Gates}

The testing of a CMOS gates and circuits is done by using the Cadence-Virtuoso tool under the GPDK $180 \mathrm{~nm}$ technology and the finger width of the transistor is fixed at $2 \mu \mathrm{m}$ similarly the finger width is also $2 \mu \mathrm{m}$ ranges.

\subsection{NOT Gate}

Trig01 is implemented in the NOT gate transistor level model. This circuit diagram explains how the design is implemented in the standard CMOS gate. By using the trigger pulse to pMOS and nMOS the operation is verified. The output is drive directly from the transistors. Operating voltage is given to the circuit is divided into a two parts by nMOS and pMOS transistor and given to the drive unit.

So that depending on the BTC signal the operating power is changed, when pMOS is triggered ON and due to the pull up logic the maximum voltage is applied to the circuit and it is operates in the maximum power mode. Implementation of TRIG 01 and TRIG10 in NOT gate as shown in Figure 3 and Figure 4 it contains the two different designs which is based on the BTC unit, voltage divide and the leakage current suppressor.

Design 2: similar to the design 1 technology the BTC is placed in the ground leakage suppressor side of the circuit. This is used to block the short circuit between the operating voltage and GND, thus it reduce the leakage current of the circuit. Four type of triggering is done by pMOS alone ON, nMOS alone ON, both pMOS and nMOS ON or both OFF. For a full power operation to switch the large load the control circuitry is switched ON fully mode i.e., pMOS and nMOS both are in closed circuit. Thus the full power is given to the CMOS circuit.

\subsection{EX-OR Gate}

Trig01 is implemented in the EX-OR gate transistor level model.

As shown in the Figure 5 and Figure 6 the EX-OR gate is controlled by the Bi-Trig control circuit at the voltage divider side of the circuit. The triggering voltage given to the circuit is control by the trigger signal given to the CMOS gate. Similarly these designs are implemented in all gates and also in different CMOS circuits and the power is calculated using the Cadence tool. The average power is measured by using the tranpow in the browser window similarly the total power consumption is done by using the cadence-average (get data). By this the overall power is tabulated and checked.

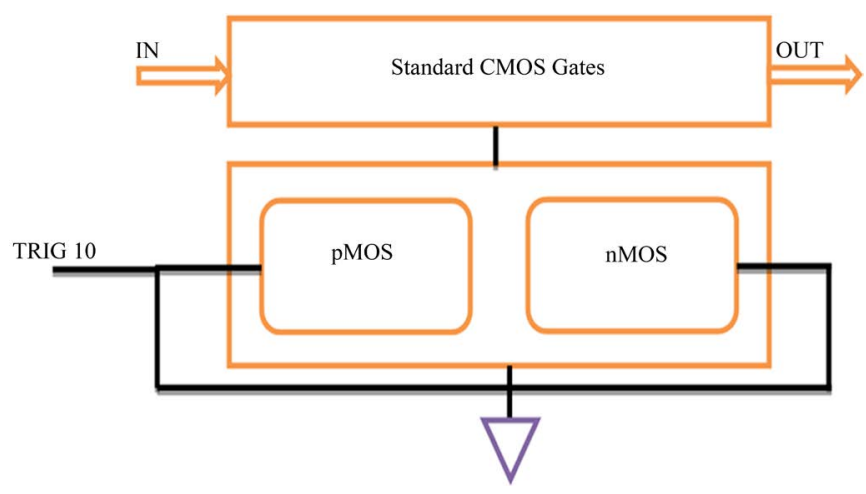

Figure 2. Trig10 block diagram. 


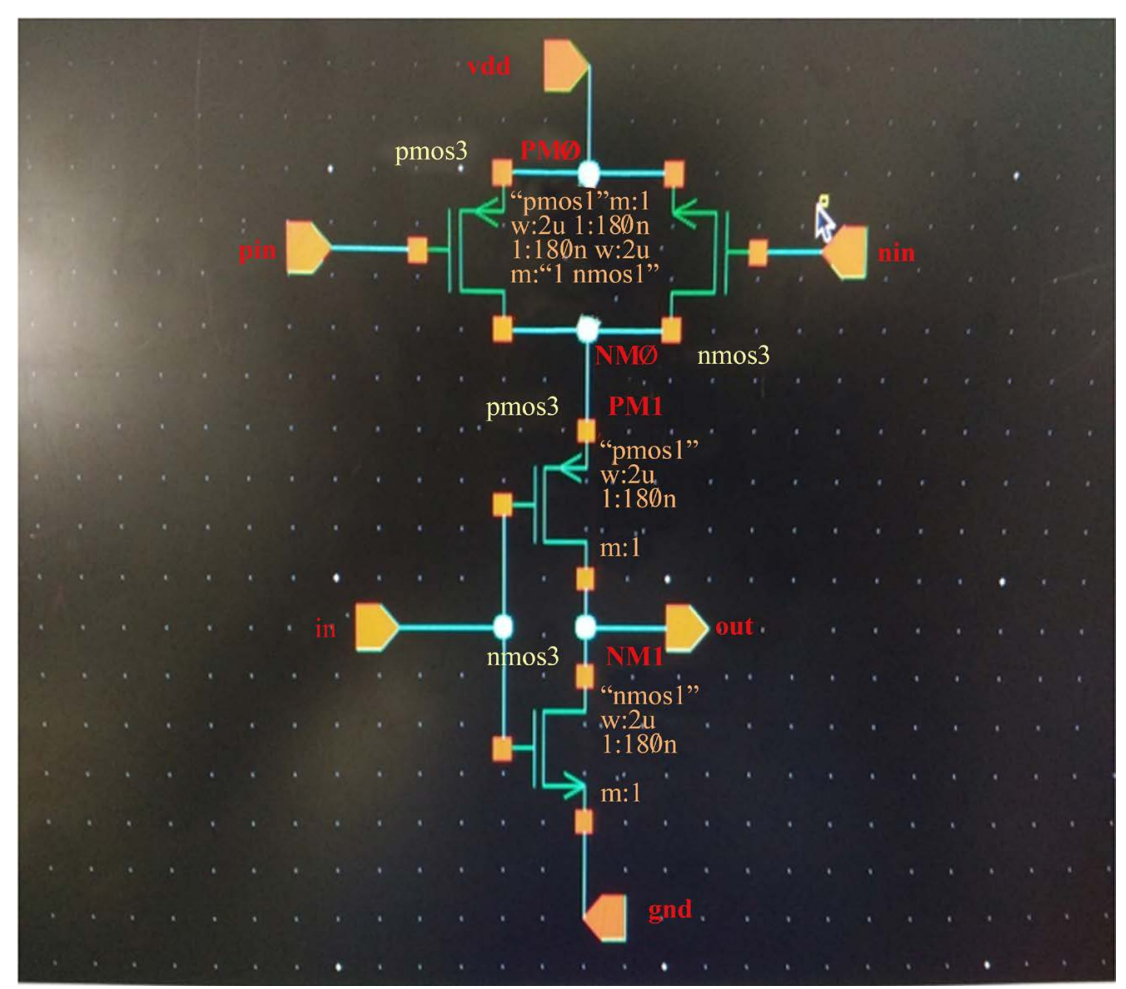

Figure 3. NOT gate with Trig01.

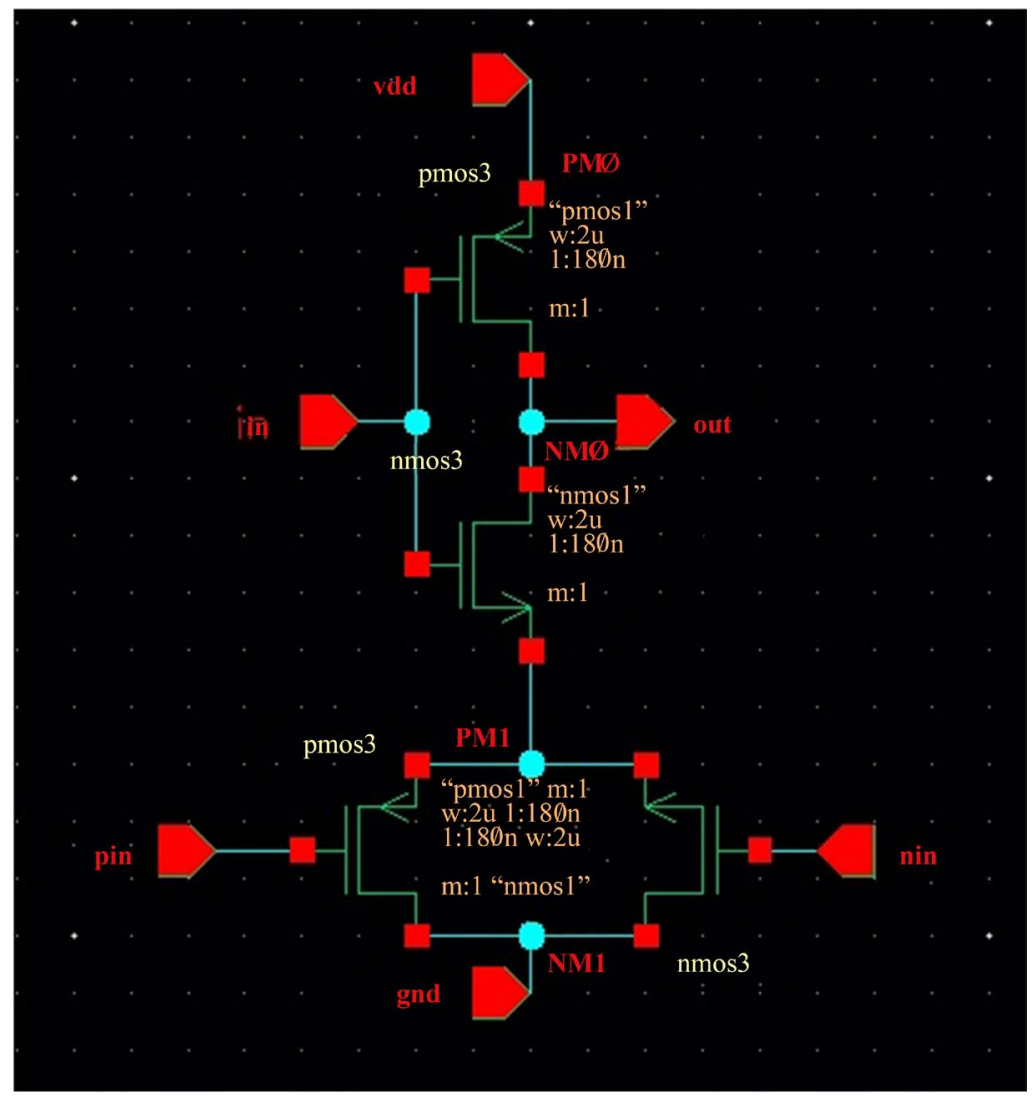

Figure 4. NOT gate with Trig10. 


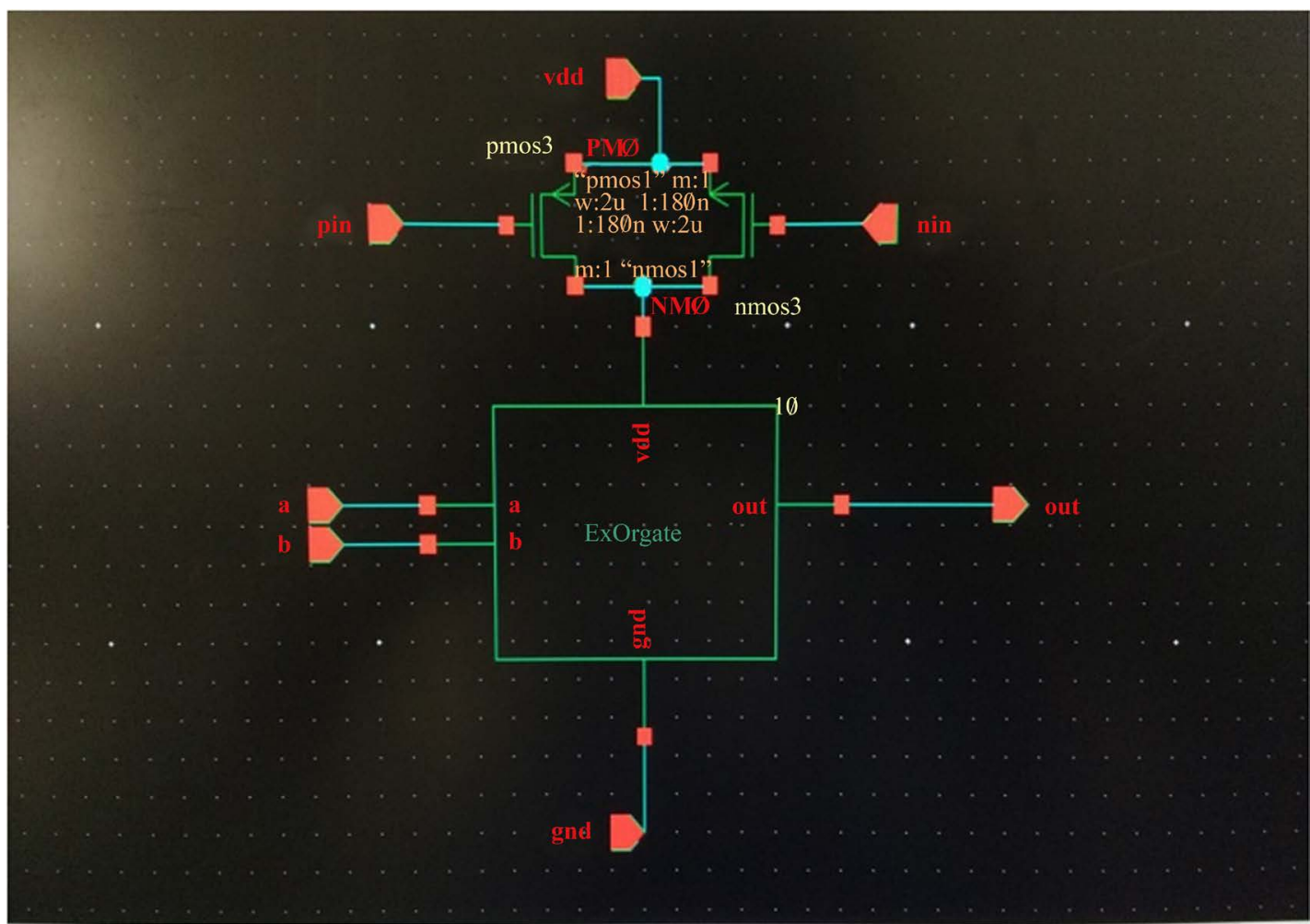

Figure 5. EX-OR gate with Trig01.

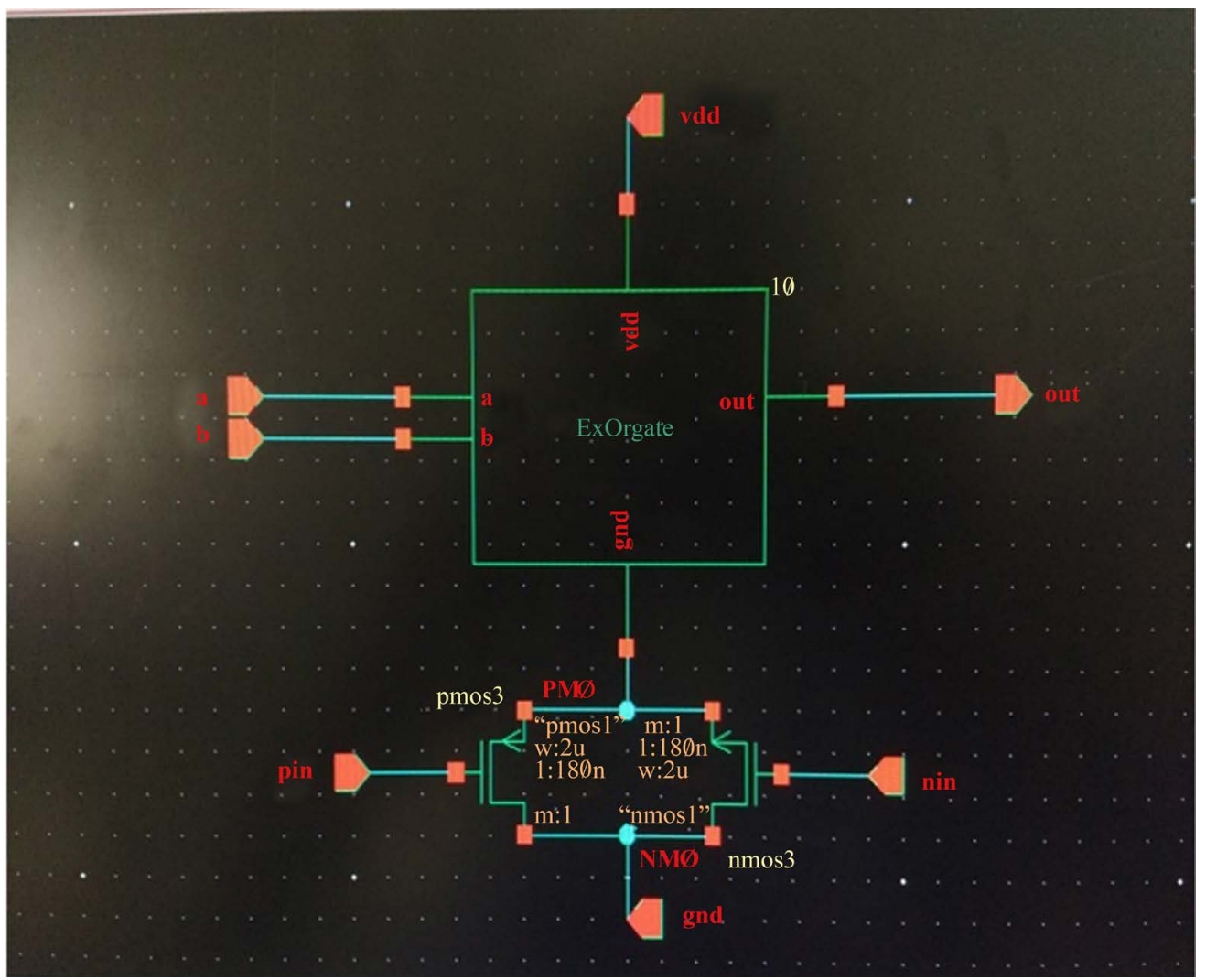

Figure 6. EX-OR gate with Trig10.

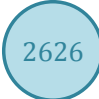




\section{Power Outputs of CMOS Gates}

The CMOS gates are designed using the BTC method and the power is calculated using the cadence-virtuoso tools calculator unit. The average power consumed by the gates under BTC is tabulated and shown in the Table 1. The power consumption will be in the range of micro wattage, BTC reduces the power wastage and also the error or noise current flow in the circuit.

\section{Implementation of the Design in CMOS Circuits Gates}

To implementing the BTC circuits the CMOS gates which is designed earlier is used with the same Trig01 and Trig10 technique. Thus the power consumption of the circuit is determined by using the group of gates work with the predefined techniques. Designing a BTC circuit the basic gates which is designed with the BTC unit is used to analyze the performance (i.e.,) power consumption of the BTC based CMOS circuit.

\subsection{Full Adder Design Using BTC}

Full adder contain a 3 in pins (ex: a, b, cin) and 2 out pins (ex:sum, carry). By using the BTC other two inputs are used (ex: pin, nin) as shown in Figure 7 and Figure 8. Here the full adder is designed with the two EX-OR gates and three AND gate and also with two numbers of the OR gate which is designed in BTC technique.

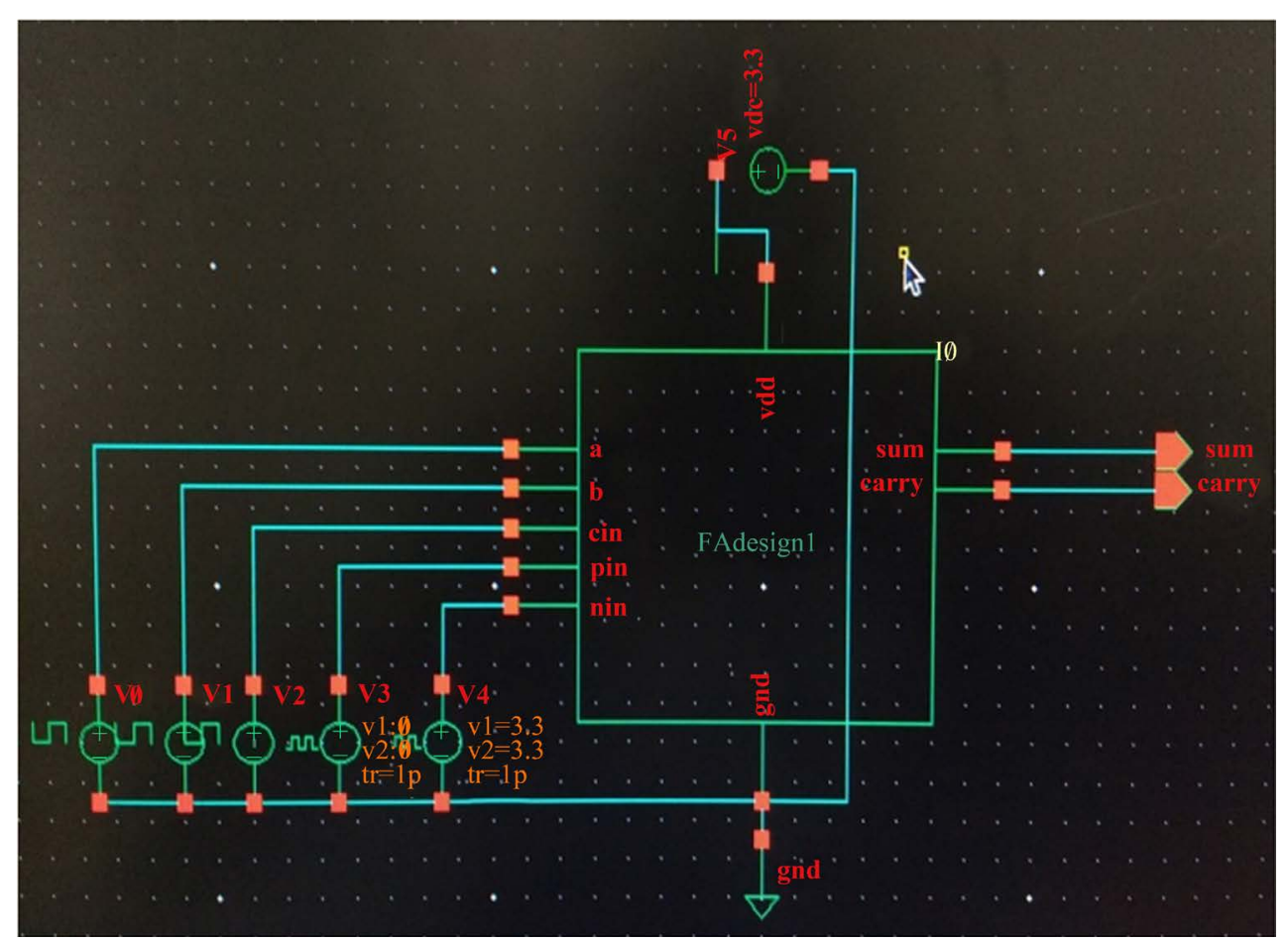

Figure 7. Full adder with BTC Trig01 gates.

Table 1. Power analysis of CMOS gates under Bi-Triggering method.

\begin{tabular}{cccc}
\hline S. NO & CMOS gates & Design 1: Power in micro watts & Design 2: Power in micro watts \\
\hline 1 & NOT gate & 1332 & 130.82 \\
2 & EX-OR gate & 107.13 & 156.92 \\
3 & AND gate & 118.92 & 145.7 \\
4 & OR gate & 87 & 134.4 \\
5 & NOR gate & 76.72 & 96.99 \\
6 & NAND gate & 111.32 & 141.7 \\
\hline
\end{tabular}




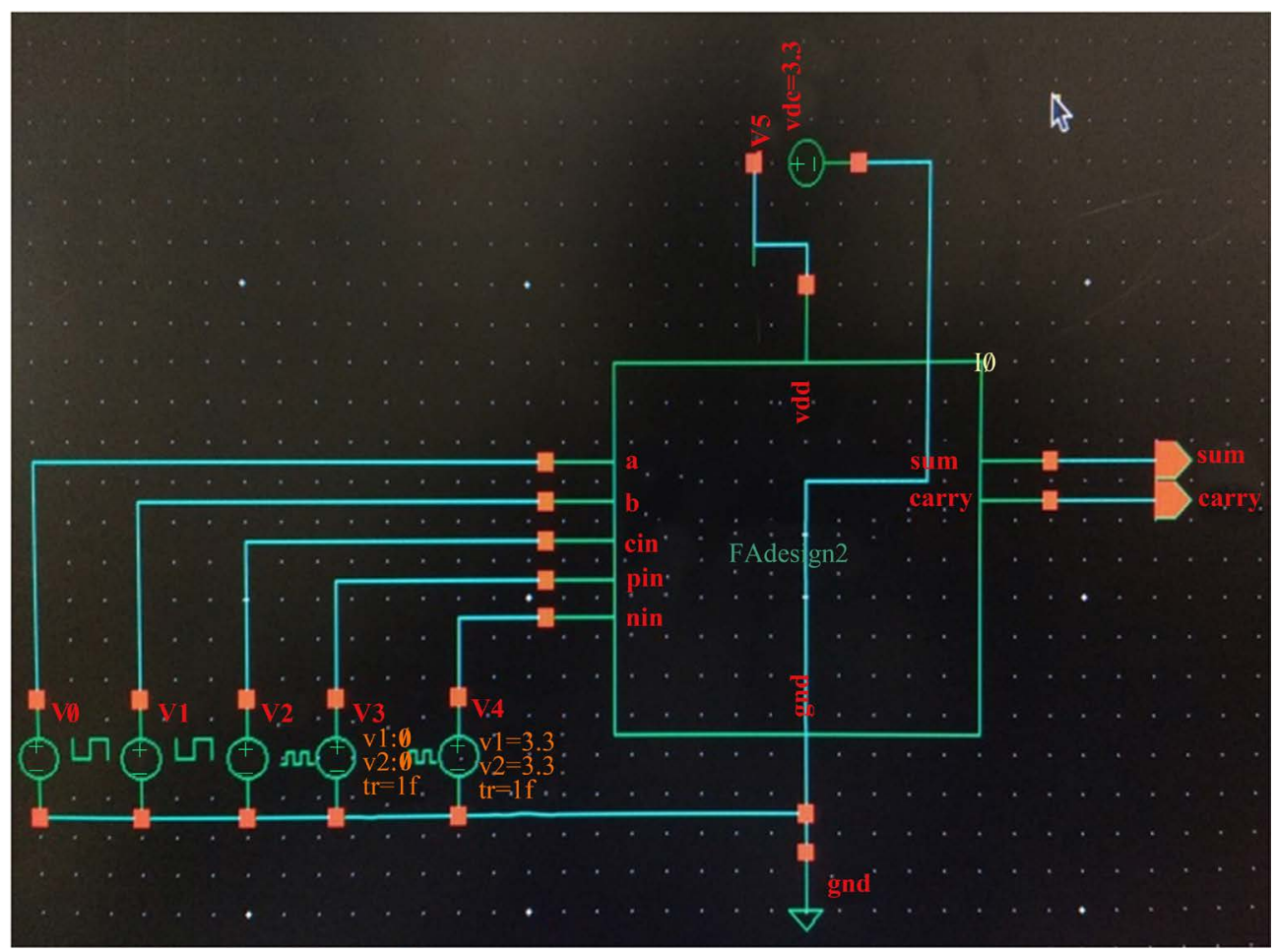

Figure 8. Full adder with BTC Trig10 gates.

The full adder designed in the papers [7]-[13] are depend only on the sizing of the transistor, reducing the finger width of the transistor due to this the circuit consume less power, even the power reduction is done due to the low width the circuit is not used in the complex design. To avoid these drawbacks the BTC is used in the two type of methodology as shown Figure 7 and Figure 8. In the paper [14] [15] the transistor operated in near threshold region thus it causes an error in the output signal to drive the next or large load.

\subsection{Carry Look Ahead Adder Design Using BTC}

Here the CLAA is designed using the BTC design 2 gates as shown in the Figure 9. The BTC of all the gates are combined in a single lined and input is switched as same. Depending on the switching the operating power is changed. Trig01 and Trig10 of this circuit is used to reduce the power consumption during ideal and operation mode.

1) Ripple Carry Adder design using BTC

RCA is designed with Full adder which is designed earlier using the Trig01 and Trig10 gates, here the cells are designed by using the BTC gates and implemented with either design 1 or design 2 logic as shown in Figure 10.

This is a 4 bit RCA unit developed using the Trig01 method. Instead of having an eight number of input It Contain the ten numbers of inputs including the BTC pins. As shown in Figure 10 and Figure 11 gives the detail pin of RCA.

\section{Parameters Comparison}

In the proposed system the CMOS circuit are designed and the some parameters are analyzed it is shown in the tables. Table 2 provides the power analysis, Table 3 provides the energy consumption of the circuits, Table 4 provides the delay analysis.

\section{Conclusion}

Thus the CMOS gates are designed using the design-1 and design-2 approach under Sub-Clocking method. These gates are used as building blocks for the development of the Sub-Clock CMOS circuits in order to reduce 


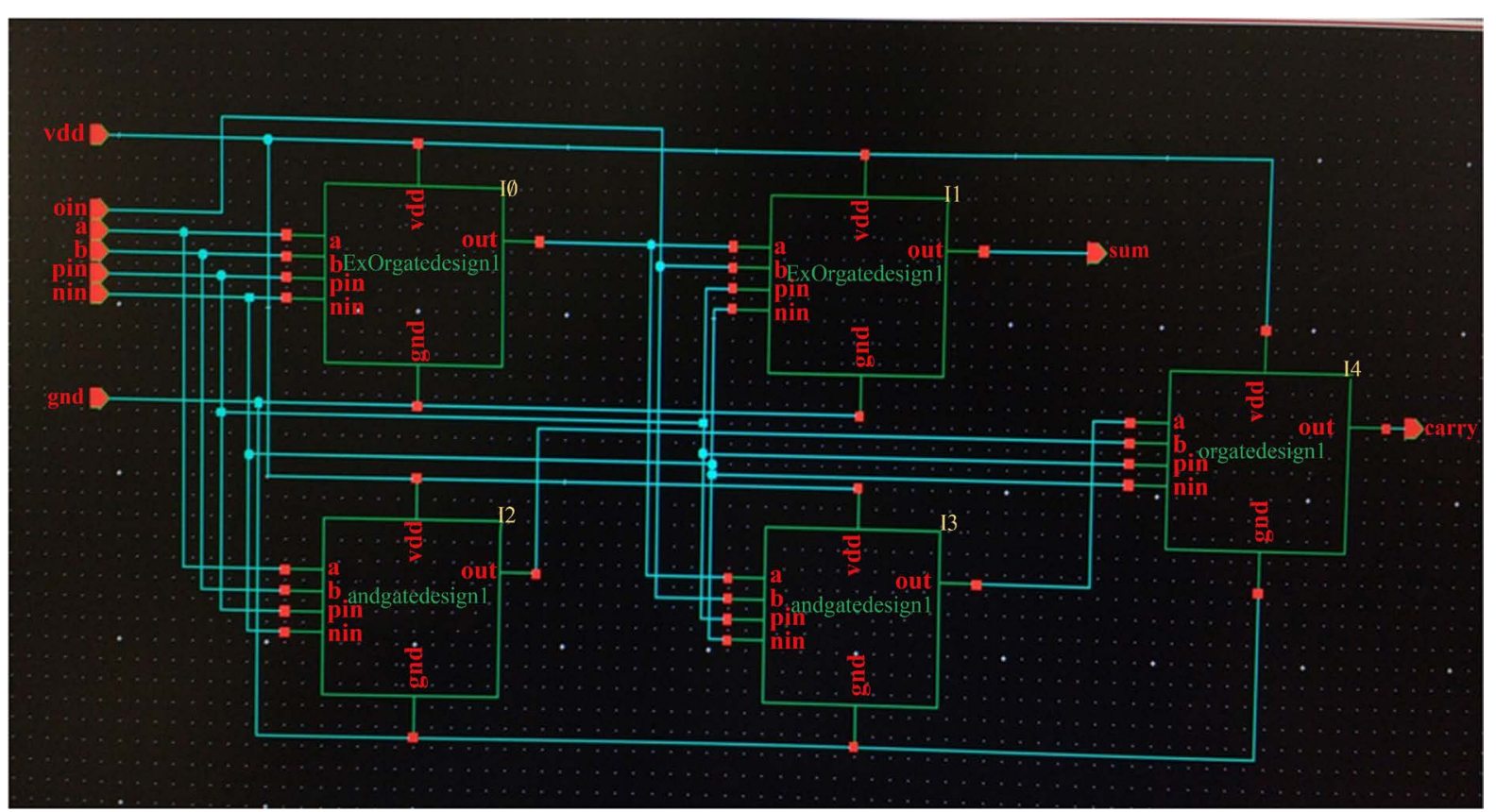

Figure 9. CLAA with BTC Trig01 gates.

Table 2. Power analysis of CMOS gates and various CMOS circuits. This comparison Table 1 gives a detail about the power consumed by the device in previous method and the proposed method. As a result nearly $50 \%$ power consumption is reduced then existing one.

\begin{tabular}{|c|c|c|c|c|}
\hline S. NO & CMOS Circuit & $\begin{array}{l}\text { Existing Design } \\
\text { power in } \mu \text { watts }\end{array}$ & $\begin{array}{l}\text { Proposed Design-1 } \\
\text { Power in } \mu \text { watts }\end{array}$ & $\begin{array}{l}\text { Proposed Design-2 } \\
\text { power in } \mu \text { watts }\end{array}$ \\
\hline 1 & NOT Gate & 28.84 & 15.31 & 13.46 \\
\hline 2 & AND Gate & 12.44 & 6.45 & 6.21 \\
\hline 3 & OR Gate & 17.21 & 9.310 & 9.11 \\
\hline 4 & NAND Gate & 10.59 & 5.630 & 5.22 \\
\hline 5 & NOR Gate & 10.43 & 6.151 & 6.03 \\
\hline 6 & EX-OR Gate & 34.50 & 18.34 & 18.40 \\
\hline 7 & Full Adder & 1215 & 79.81 & 78.56 \\
\hline 8 & Carry Look Ahead Adder & 915.30 & 66.79 & 67.69 \\
\hline 9 & Ripple Carry Adder & 375.00 & 247.40 & 176.1 \\
\hline 10 & Carry Select Adder & 19950 & 13170 & 10650 \\
\hline 11 & D Flip Flop & 216.06 & 21.40 & 38.78 \\
\hline 12 & SR Latch & 10.92 & 7.917 & 7.723 \\
\hline 13 & Shift Register & 610.56 & 410.90 & 390.5 \\
\hline 14 & Novel Low Power FA 180 nm & 76.73 & 47.48 & 44.96 \\
\hline 15 & Novel Low Power FA 90 nm & 3.003 & 1.731 & 1.722 \\
\hline 16 & Novel Low Power FA 45 nm & 0.501 & 0.383 & 0.375 \\
\hline 17 & Peres gate & 61.48 & 36.79 & 36.47 \\
\hline 18 & Feynman Gate & 49.66 & 34.36 & 33.51 \\
\hline 19 & Fredkin Gate & 115.50 & 67.56 & 66.01 \\
\hline 20 & C17-ISCAS85 & 72.68 & 63.29 & 58.07 \\
\hline 21 & S27 & 3270.00 & 309.40 & 515.0 \\
\hline
\end{tabular}




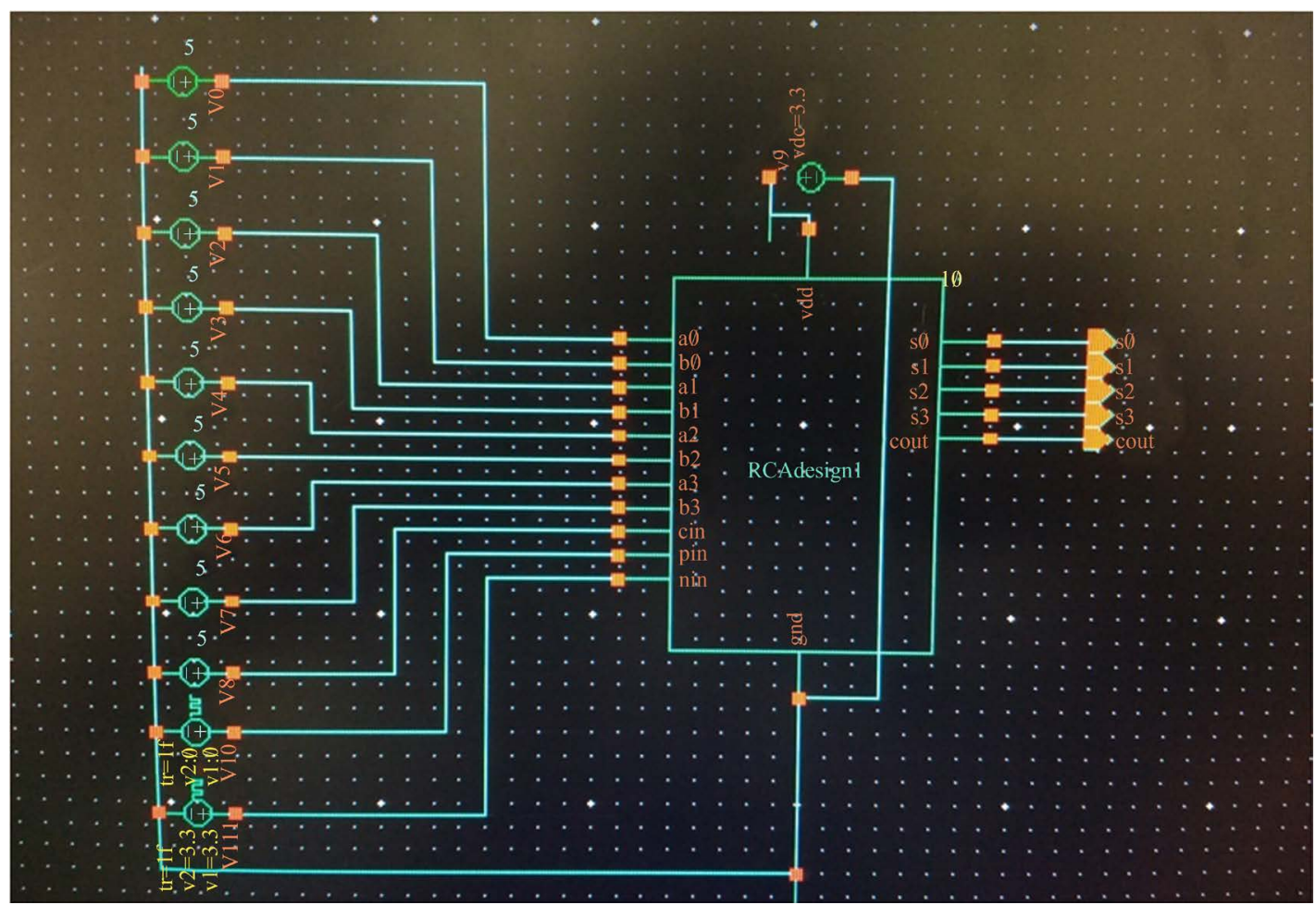

Figure 10. Ripple carry adder with Trig01 gates.

Table 3. Energy analysis of CMOS gates and various CMOS circuits. This comparison Table 2 gives a detail about the energy consumed by the device in previous method and the proposed method. As a result nearly $48 \%$ energy consumption is reduced then existing one.

\begin{tabular}{ccccc}
\hline S. NO & CMOS Circuit & $\begin{array}{c}\text { Existing Design } \\
\text { Energy in nano joules }\end{array}$ & $\begin{array}{c}\text { Proposed Design-1 } \\
\text { Energy in nano joules }\end{array}$ & $\begin{array}{c}\text { Proposed Design-2 } \\
\text { Energy in nano joules }\end{array}$ \\
\hline 1 & NOT Gate & 2.88 & 1.50 & 1.36 \\
2 & AND Gate & 0.490 & 0.258 & 0.248 \\
3 & OR Gate & 0.688 & 0.372 & 0.364 \\
4 & NAND Gate & 0.423 & 0.225 & 0.208 \\
5 & NOR Gate & 0.417 & 0.246 & 0.241 \\
6 & EX-OR Gate & 1.38 & 0.733 & 6.28 \\
7 & Full Adder & 97.20 & 6.38 & 5.41 \\
8 & Carry Look Ahead Adder & 73.20 & 5.34 & 14.08 \\
9 & Ripple Carry Adder & 30.00 & 19.79 & 42.60 \\
10 & Carry Select Adder & 79.80 & 52.68 & 3.87 \\
11 & D Flip Flop & 21.60 & 2.14 & 0.308 \\
12 & SR Latch & 0.436 & 0.316 & 39.05 \\
13 & Shift Register & 61.00 & 41.09 & 3.59 \\
14 & Novel Low Power FA 180 nm & 6.13 & 3.79 & 0.137 \\
15 & Novel Low Power FA 90 nm & 0.240 & 0.138 & 0.030 \\
16 & Novel Low Power FA 45 nm & 0.040 & 0.030 & 1.45 \\
17 & Peres gate & 2.45 & 1.47 & 1.34 \\
18 & Feynman Gate & 1.98 & 1.37 & 5.28 \\
19 & Fredkin Gate & 9.24 & 5.40 & 5.80 \\
20 & C17-ISCAS85 & 7.20 & 6.32 & 41.2 \\
\hline
\end{tabular}




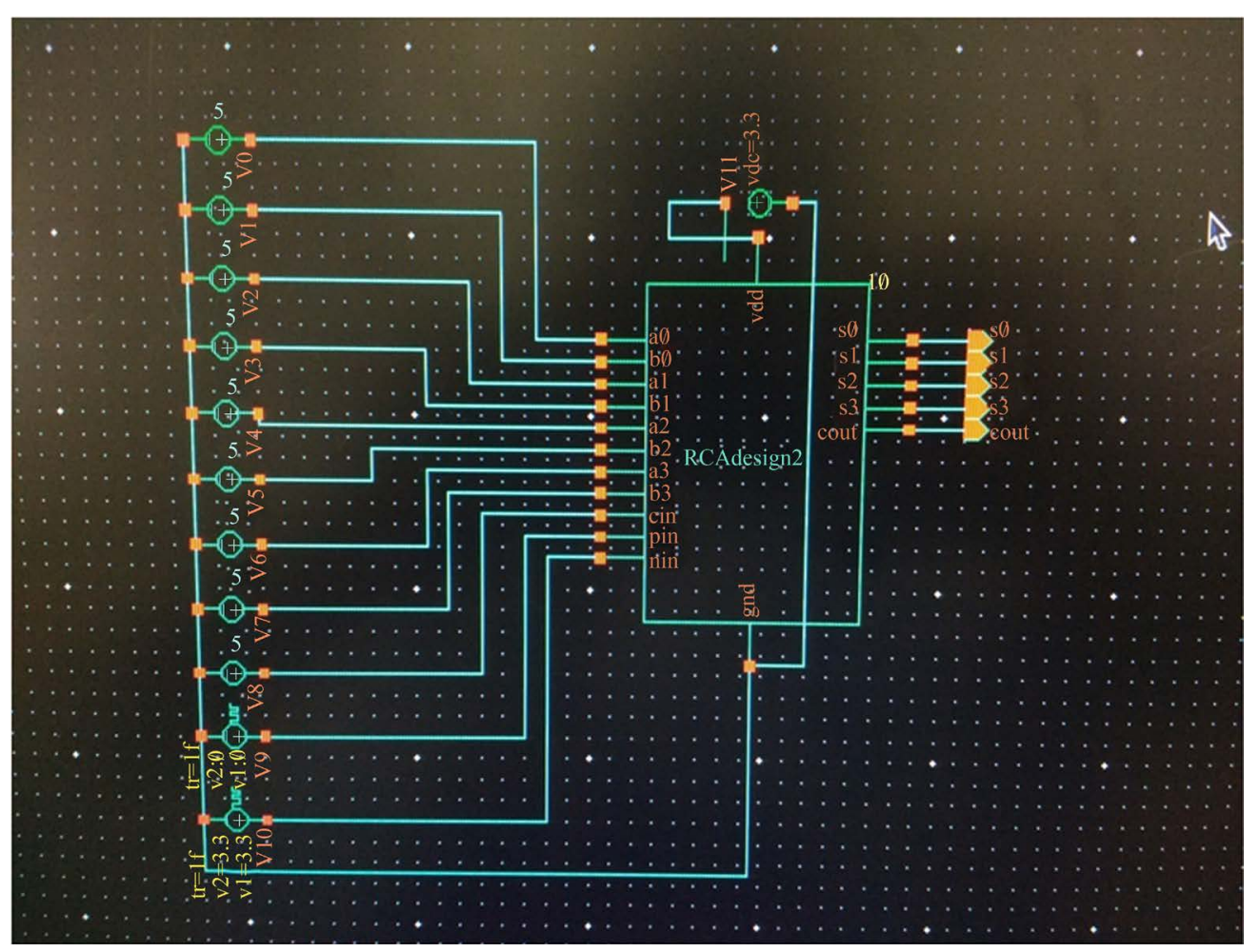

Figure 11. Ripple carry adder with Trig10 gates.

Table 4. Delay analysis of CMOS gates and various CMOS circuits. This comparison Table 3 gives a detail about the maximum delay by the device in previous method and the proposed method. As a result delays are same as in the previous method even transistor are added in additional.

\begin{tabular}{ccccc}
\hline S.NO & CMOS Circuit & $\begin{array}{c}\text { Existing Design } \\
\text { Delay in Pico seconds }\end{array}$ & $\begin{array}{c}\text { Proposed Design-1 } \\
\text { Delay in Pico seconds }\end{array}$ & $\begin{array}{c}\text { Proposed Design-2 } \\
\text { Delay in Pico seconds }\end{array}$ \\
\hline 1 & NOT Gate & 26.56 & 25.98 & 26.02 \\
2 & AND Gate & 20.06 & 20.06 & 20.06 \\
3 & OR Gate & 40.01 & 39.21 & 39.85 \\
4 & NAND Gate & 20.05 & 20.05 & 20.05 \\
5 & NOR Gate & 49.41 & 47.77 & 48.42 \\
6 & EX-OR Gate & 65.15 & 65.23 & 141.60 \\
7 & Full Adder & 140.30 & 140.25 & 142.88 \\
8 & Carry Look Ahead Adder & 142.90 & 142.79 & 139.12 \\
9 & Ripple Carry Adder & 139.10 & 138.92 & 600.20 \\
10 & Carry Select Adder & 599.70 & 598.99 & 81.15 \\
11 & D Flip Flop & 81.19 & 81.02 & 10,020 \\
12 & SR Latch & 10,020 & 10,040 & 9802 \\
13 & Shift Register & 9800 & 9788 & 81.02 \\
14 & Novel Low Power FA 180 nm & 80.71 & 80.59 & 41.03 \\
15 & Novel Low Power FA 90 nm & 40.92 & 40.82 & 14.20 \\
16 & Novel Low Power FA 45 nm & 14.31 & 14.19 & 36.32 \\
17 & Peres Gate & 36.29 & 36.19 & 36.22 \\
18 & Feynman Gate & 36.09 & 36.09 & 10,072 \\
19 & Fredkin Gate & 10,070 & 10,069 & 10,051 \\
20 & C17-ISCAS85 & 10,050 & 10,051 & 30,025 \\
21 & S27 & 30,020 & 30,022 & \\
\hline
\end{tabular}


the power consumption and also the leakage current. The power consumed by the sub-clock circuit is only about 46\% when compared to the ideal circuit [1]-[7]. Similarly the energy consumed by the circuit is also reduced up to $40 \%$ compared to the existing/ideal circuits. This method does not affect the performance of the circuit; delay of the circuit is similar to that of the ideal CMOS circuit and produces about $95 \%$ of efficiency. This can be implemented in any kind of logic in digital circuit. These designs can be extended to dual sleep technique and also in dual stack technique in order to utilize the whole circuit performance. Sub-clocking is the technique which is more efficient than that of the Dual Mode Logic [9] and an ideal circuit without sub-clock method. Depending on the technology $(180 \mathrm{~nm}, 90 \mathrm{~nm}, 45 \mathrm{~nm})$ and period of operation, the energy consumption can be varied and also the power consumption is reduced up to 54\% [11]. To get the proper operating point, the width of the transistor is varied using the Cadence Virtuoso tool.

\section{References}

[1] Kaizerman, A., Fisher, S. and Fish, A. (2013) Subthreshold Dual Mode Logic. IEEE Transactions on Very Large Scale Integration System, 21, 979-983. http://dx.doi.org/10.1109/TVLSI.2012.2198678

[2] Bol, D., Ambroise, R., Flandre, D. and Legat, J.D. (2008) Analysis and Minimization of Practical Energy in $45 \mathrm{~nm}$ Subthreshold Logic Circuits. IEEE International Conference on Computer Design, Lake Tahoe, 12-15 October 2008, 294-300.

[3] Calhoun, B.H., Wang, A. and Chandrakasan, A. (2005) Modeling and Sizing for Minimum Energy Operation in Subthreshold Circuits. IEEE Journal of Solid-State Circuits, 40, 1778-1786. http://dx.doi.org/10.1109/JSSC.2005.852162

[4] Alioto, M. (2012) Ultralow Power VLSI Circuit Design Demystified and Explained: A Tutorial. IEEE Transactions on Circuits Systems I, 59, 3-29. http://dx.doi.org/10.1109/TCSI.2011.2177004

[5] Chang, M.H, Hsieh, C.Y., Chen, M.W. and Hwang, W. (2015) Logical Effort Models with Voltage and Temperature Extensions in Super-/Near-/Sub-Threshold Regions. 2011 International Symposium on VLSI Design, Automation and Test (VLSI-DAT), Hsinchu, 25-28 April 2011, 1-4.

[6] Kwong, J. and Chandrakasan, A.P. (2006) Variation-Driven Device Sizing for Minimum Energy Sub-Threshold Circuits. Proceedings of the 2006 International Symposium on Low Power Electronics and Design, Bavaria, 4-6 October 2006, 8-13. http://dx.doi.org/10.1145/1165573.1165578

[7] Levi, Kaizerman, A. and Fish, A. (2012) Low Voltage Dual Mode Logic: Model Analysis and Parameter Extraction Excepted Elsevier. Microelectronics Journal, 12, 1778-1786.

[8] Liu, X., Zheng, Y., Phyu, M.W., Endru, F.W., Navaneethan, V. and Zhao, B. (2012) An Ultra-Low Power ECG Acquisition and Monitoring ASIC System for WBAN Applications. IEEE Journal on Emerging and Selected Topics in Circuits and Systems, 2, 60-70. http://dx.doi.org/10.1109/JETCAS.2012.2187707

[9] Markovic, D., Wang, C.C., Alarcon, L.P. and Rabaey, J.M. (2010) Ultralow Power Design in Near-Threshold Region. Proceedings of the IEEE, 98, 237-252. http://dx.doi.org/10.1109/JPROC.2009.2035453

[10] Morgenshtein, E.G., Friedman, Ginosar, R. and Kolodny, A. (2010) Unified logical Effort-A Method for Delay Evaluation and Minimization in Logic Paths with Interconnect. IEEE Transactions on Very Large Scale Integration (VLSI) Systems, 18, 689-696. http://dx.doi.org/10.1109/TVLSI.2009.2014239

[11] Pu, Y, de Gyvez, J.P. and Corporaal, H. (2009) An Ultralowenergy/Frame Multi-Standard JPEG Co-Processor in 65 nm CMOS with Sub/Near-Threshold Power Supply. 2009 IEEE International Solid-State Circuits Conference, San Francisco, 8-12 February 2009, 146-147.

[12] Razak, H. (2008) High Performance ASIC Design: Using Synthesizable Domino Logic in an ASIC Flow. Cambridge University, Cambridge.

[13] Soeleman, H., Roy, K. and Paul, B. (2001) Sub-Domino Logic: Ultralow Power Dynamic Sub-Threshold Digital Logic. Fourteenth International Conference on VLSI Design, Bangalore, 7 January 2001, 211-214. http://dx.doi.org/10.1109/icvd.2001.902662

[14] Verma, N., Kwong, J. and Chandrakasan, A. (2008) Nanometer MOSFET Variation in Minimum Energy Subthreshold Circuits. IEEE Transactions on Electron Devices, 55, 163-174. http://dx.doi.org/10.1109/TED.2007.911352

[15] Yamada, H., Hotta, T., Nishiyama, T., Murabayashi, F. and Yamauchi, T. (1995) A 13.3 ns Double-Precision FloatingPoint ALU and Multiplier. 1995 IEEE International Conference on Computer Design: VLSI in Computers and Processors, 2-4 October 1995, 466-470. 


\section{Submit or recommend next manuscript to SCIRP and we will provide best service for you:}

Accepting pre-submission inquiries through Email, Facebook, LinkedIn, Twitter, etc.

A wide selection of journals (inclusive of 9 subjects, more than 200 journals)

Providing 24-hour high-quality service

User-friendly online submission system

Fair and swift peer-review system

Efficient typesetting and proofreading procedure

Display of the result of downloads and visits, as well as the number of cited articles

Maximum dissemination of your research work

Submit your manuscript at: http://papersubmission.scirp.org/ 